\title{
Deep Learning for the Preoperative Diagnosis of Metastatic Cervical Lymph Nodes on Contrast-Enhanced Computed ToMography in Patients with Oral Squamous Cell Carcinoma
}

\author{
Hayato Tomita $1,2, * \mathbb{D}$, Tsuneo Yamashiro ${ }^{2}\left(\mathbb{D}\right.$, Joichi Heianna ${ }^{2}\left(\mathbb{D}\right.$, Toshiyuki Nakasone ${ }^{3}$, Tatsuaki Kobayashi ${ }^{4}(\mathbb{D}$, \\ Sono Mishiro ${ }^{5}$, Daisuke Hirahara ${ }^{5}{ }^{\circ}$, Eichi Takaya ${ }^{6}{ }^{\circ}$, Hidefumi Mimura ${ }^{1}$, Sadayuki Murayama ${ }^{2}$ and \\ Yasuyuki Kobayashi ${ }^{4}$
}

Citation: Tomita, H.; Yamashiro, T.; Heianna, J.; Nakasone, T.; Kobayashi, T.; Mishiro, S.; Hirahara, D.; Takaya, E.; Mimura, H.; Murayama, S.; et al. Deep Learning for the Preoperative Diagnosis of Metastatic Cervical Lymph Nodes on Contrast-Enhanced Computed ToMography in Patients with Oral Squamous Cell Carcinoma. Cancers 2021, 13, 600. https:// doi.org/10.3390/cancers13040600

Academic Editors: Khaled M. Elsayes and Tanya W. Moseley

Received: 22 December 2020

Accepted: 31 January 2021

Published: 3 February 2021

Publisher's Note: MDPI stays neutral with regard to jurisdictional claims in published maps and institutional affiliations.

Copyright: (c) 2021 by the authors. Licensee MDPI, Basel, Switzerland. This article is an open access article distributed under the terms and conditions of the Creative Commons Attribution (CC BY) license (https:/ / creativecommons.org/licenses/by/ $4.0 /)$.
1 Department of Radiology, St. Marianna University School of Medicine, 2-16-1 Sugao, Miyamae-ku, Kawasaki, Kanagawa 216-8511, Japan; mimura@marianna-u.ac.jp

2 Department of Radiology, Graduate School of Medical Science, University of the Ryukyus, 207 Uehara, Nishihara, Okinawa 903-0215, Japan; clatsune@yahoo.co.jp (T.Y.); anna1150@med.u-ryukyu.ac.jp (J.H.); sadayuki@med.u-ryukyu.ac.jp (S.M.)

3 Department of Oral and Maxillofacial Surgery, Graduate School of Medical Science, University of the Ryukyus, 207 Uehara, Nishihara, Okinawa 903-0215, Japan; nakasone4266@gmail.com

4 Department of Advanced Biomedical Imaging Informatics, St. Marianna University School of Medicine, 2-16-1 Sugao, Miyamae-ku, Kawasaki, Kanagawa 216-8511, Japan; t_kobayashi@vis-ionary.com (T.K.); yasukoba2@gmail.com (Y.K.)

5 Department of AI Research Lab, Harada Academy, 2-54-4 Higashitaniyama, Kagoshima, Kagoshima 891-0113, Japan; mishiro@harada-gakuen.ac.jp (S.M.); rt.hirahara@harada-gakuen.ac.jp (D.H.)

6 School of Science for Open and Environmental Systems, Graduate School of Science and Technology, Keio University, 3-14-1 Hiyoshi, Kohoku-ku, Yokohama, Kanagawa 223-8522, Japan; etakaya@keio.jp

* Correspondence: m04149@yahoo.co.jp; Tel.: +81-44-977-8111

Simple Summary: Cervical lymph node (LN) metastasis in patients with oral squamous cell carcinoma is one of the important prognostic factors. Pretreatment cervical nodal staging is performed using computed tomography (CT) as the first-line examination. However, imaging findings focused on morphology are not specific for detecting cervical LN metastasis. In this study, deep learning (DL) analysis of pretreatment contrast-enhanced CT was evaluated and compared with radiologists' assessments at levels I-II, I, and II using the independent test set. The DL model achieved higher diagnostic performance in discriminating between benign and metastatic cervical LNs at levels I-II, I, and II. Significant difference in the area under the curves of the DL model and the radiologists assessments at levels I-II and II were observed. Our findings suggest that this approach can provide additional value to treatment strategies.

Abstract: We investigated the value of deep learning (DL) in differentiating between benign and metastatic cervical lymph nodes (LNs) using pretreatment contrast-enhanced computed tomography (CT). This retrospective study analyzed 86 metastatic and 234 benign (non-metastatic) cervical LNs at levels I-V in 39 patients with oral squamous cell carcinoma (OSCC) who underwent preoperative CT and neck dissection. LNs were randomly divided into training $(70 \%)$, validation $(10 \%)$, and test $(20 \%)$ sets. For the validation and test sets, cervical LNs at levels I-II were evaluated. Convolutional neural network analysis was performed using Xception architecture. Two radiologists evaluated the possibility of metastasis to cervical LNs using a 4-point scale. The area under the curve of the DL model and the radiologists' assessments were calculated and compared at levels I-II, I, and II. In the test set, the area under the curves at levels I-II (0.898) and II (0.967) were significantly higher than those of each reader (both, $p<0.05$ ). DL analysis of pretreatment contrast-enhanced CT can help classify cervical LNs in patients with OSCC with better diagnostic performance than radiologists' assessments alone. DL may be a valuable diagnostic tool for differentiating between benign and metastatic cervical LNs. 
Keywords: deep learning; cervical lymph node; convolutional neural network; level; squamous cell carcinoma

\section{Introduction}

Metastasis to the cervical lymph nodes (LNs) is one of the poor prognostic factors in patients with oral squamous cell carcinoma (OSCC). Evaluating whether the cervical LNs are benign or metastatic depends on the treatment strategy. Among patients with clinically negative LNs, $15-20 \%$ are at risk of occult LN metastasis [1]. Unnecessary surgical LN dissection without metastatic cervical LNs can lead to increased complications, while delayed dissection of LN metastases can result in disease progression. Ultrasonography (US), computed tomography (CT), magnetic resonance imaging (MRI), and fluorine-18-2fluoro-2-deoxy-D-glucose positron emission tomography (18F-FDG PET) have been widely used for evaluating the cervical LN status in head and neck cancer patients [2-4]. However, the subjective nature of the morphologic criteria for visually confirming metastatic LNs on US, CT, and MRI results in diminished reproducibility and objectivity. Although several studies have recently described the usefulness of dual-energy $\mathrm{CT}$ to evaluate the cervical LN status in head and neck cancer patients, it is not widely used [5,6]. 18F-FDG PET has been known to be the best modality for evaluating cervical LN metastasis in these patients. However, the diagnosis of small cervical LNs for evaluating the nodal status using 18F-FDG PET is limited, owing to false-negative findings [7,8]. Additionally, the sensitivity of sentinel LN biopsy and sentinel LN imaging techniques using CT or MR lymphography and PET lymphoscintigraphy is $56-91 \%$ [9,10]. Unfortunately, metastatic cervical LNs are not easily detected on a pretreatment clinical examination. Therefore, the development of accurate diagnostic methods is required.

With the continued development of artificial intelligence, deep learning (DL) has been applied to medical imaging for tissue characterization, outcome prediction, and automated detection [11-15]. DL enables the parameters to increase and handle complex tasks by increasing the layers of the neural networks that imitate models of brain structures connecting a large number of neurons. Convolutional neural network (CNN), one of the DL architectures, consists of convolutional and pooling layers. Convolutional layers convert some pixels in the grid into one pixel and extract the image features called a feature map. Pooling layers decrease the amount of calculation and adapt to the misalignment of images by reducing the data of the feature map. CNNs can play an important role in interpreting medical imaging without subjective assessment. Previous studies have shown how $\mathrm{CNN}$ could effectively assess the malignancy of hepatocellular carcinoma and prostate cancer lesions $[13,14]$. Furthermore, DL was able to help discriminate between benign and metastatic cervical LNs in patients with OSCC [16]. However, its value based on the American Head and Neck Society cervical regional lymph node level system, which has been used to determine the extent of LN dissection and indication for radiotherapy, has not been evaluated. Although LNs at levels I and II are known to drain from the lymphatic tract of the OSCC, identifying metastatic cervical LNs remains challenging because of oral and sinonasal inflammation or insufficient malignant deposits. In addition, prophylactic cervical neck dissection is frequently performed level-by-level in clinical practice since cervical LNs metastasis in OSCC can occur even if these are clinically diagnosed as benign lesions. Hence, unnecessary neck dissection can be prevented if the benign or metastatic LNs can be distinguished for each level. Therefore, we aimed to clarify the diagnostic performance of DL in differentiating between benign and metastatic level I-II, I, and II cervical LNs on contrast-enhanced CT in patients with OSCC.

\section{Results}

Patient characteristics are summarized in Table 1. Table 2 shows the number of LNs at each level in each set. 
Table 1. Patient-based characteristics.

\begin{tabular}{|c|c|c|}
\hline \multicolumn{2}{|c|}{ Characteristics } & $\begin{array}{c}\text { Patient-Based } \\
n=39\end{array}$ \\
\hline Age mean $+\mathrm{SD}$ & & $640+140$ \\
\hline Gender male/female & & $23 / 16$ \\
\hline \multirow[t]{3}{*}{ Primary tumor sites } & Oral tongue & 26 \\
\hline & Gingiva & 8 \\
\hline & Floor of mouse & 5 \\
\hline \multirow[t]{4}{*}{ T stage } & $\mathrm{T} 1$ & 7 \\
\hline & $\mathrm{T} 2$ & 17 \\
\hline & $\mathrm{T} 3$ & 7 \\
\hline & $\mathrm{T} 4$ & 8 \\
\hline \multirow{7}{*}{ N stage } & No & 7 \\
\hline & N1 & 11 \\
\hline & $\mathrm{N} 2 \mathrm{a}$ & 0 \\
\hline & $\mathrm{N} 2 \mathrm{~b}$ & 13 \\
\hline & $\mathrm{N} 2 \mathrm{c}$ & 6 \\
\hline & N3a & 0 \\
\hline & $\mathrm{N} 3 \mathrm{~b}$ & 2 \\
\hline \multirow{2}{*}{\multicolumn{2}{|c|}{ Level I }} & 121 \\
\hline & & 84 \\
\hline \multicolumn{2}{|c|}{ Ipsilateral Level I } & 132 \\
\hline \multirow{2}{*}{\multicolumn{2}{|c|}{ Ipsilateral Level II }} & 72 \\
\hline & & 37 \\
\hline Level III & el III & 19 \\
\hline \multirow[t]{2}{*}{ Level IV } & & 4 \\
\hline & el IV & 4 \\
\hline \multirow[t]{2}{*}{ Level V } & & 26 \\
\hline & el V & 13 \\
\hline
\end{tabular}

Table 2. The number of lymph nodes (LN) at each level in train set, validation set, and test set.

\begin{tabular}{|c|c|c|c|c|c|c|}
\hline \multirow{3}{*}{ Level } & \multicolumn{6}{|c|}{ LN-Based } \\
\hline & \multicolumn{2}{|c|}{ Train Cohort $(n=224)$} & \multicolumn{2}{|c|}{ Validation Cohort $(n=32)$} & \multicolumn{2}{|c|}{ Test Cohort $(n=64)$} \\
\hline & $\begin{array}{c}\text { Benign } \\
(n=169)\end{array}$ & $\begin{array}{c}\text { Metastasis } \\
(n=55)\end{array}$ & $\begin{array}{l}\text { Benign } \\
(n=22)\end{array}$ & $\begin{array}{c}\text { Metastasis } \\
(n=10)\end{array}$ & $\begin{array}{l}\text { Benign } \\
(n=43)\end{array}$ & $\begin{array}{c}\text { Metastasis } \\
(n=21)\end{array}$ \\
\hline Level I & 51 & 24 & 10 & 5 & 21 & 10 \\
\hline Ipsilateral Level I & 29 & 20 & 7 & 5 & 15 & 8 \\
\hline Level II & 64 & 18 & 12 & 5 & 22 & 11 \\
\hline Ipsilateral Level II & 26 & 14 & 4 & 5 & 12 & 11 \\
\hline Level III & 27 & 10 & - & - & - & - \\
\hline Ipsilateral Level III & 12 & 7 & - & - & - & - \\
\hline Level IV & 1 & 3 & - & - & - & - \\
\hline Ipsilateral Level IV & 0 & 3 & - & - & - & - \\
\hline Level V & 26 & 0 & - & - & - & - \\
\hline Ipsilateral Level V & 13 & 0 & - & - & - & - \\
\hline
\end{tabular}

\subsection{Diagnostic Performance of the Deep Learning Model in the Validation and Test Sets}

In the validation set, the DL model achieved a diagnostic accuracy rate/area under the receiver operating characteristic curve (AUC) of 97.5\%/0.964 at levels I-II. A summary of the diagnostic performances of the DL model and the radiologists' assessments in the test set is shown in Table 3. The DL model achieved a diagnostic accuracy rate/AUC of $85.9 \% / 0.898$ at levels I-II, 83.9\%/0.824 at level I, and 90.9\%/0.967 at level II. 
Table 3. Comparisons of area under the curve (AUC), diagnostic accuracy rate, sensitivity, and specificity between deep learning models and radiologists at each level.

\begin{tabular}{|c|c|c|c|c|}
\hline & AUC [95\% Confidence Interval] & Accuracy & Sensitivity & Specificity \\
\hline \multicolumn{5}{|c|}{ Level I/II } \\
\hline Deep learning & $0.898[0.778,0.956]$ & 85.9 & 66.7 & 95.4 \\
\hline Reader 1 & $0.780[0.559,0.864]^{*}$ & 78.1 & 57.1 & 88.4 \\
\hline Reader 2 & $0.758[0.587,0.873]^{*}$ & 78.1 & 66.7 & 83.7 \\
\hline \multicolumn{5}{|c|}{ Level I } \\
\hline Deep learning & $0.824[0.600,0.936]$ & 80.6 & 60.0 & 90.5 \\
\hline Reader 1 & $0.738[0.497,0.889]$ & 77.4 & 60.0 & 85.7 \\
\hline Reader 2 & $0.707[0.443,0.880]$ & 74.2 & 60.0 & 81.0 \\
\hline \multicolumn{5}{|c|}{ Level II } \\
\hline Deep learning & $0.967[0.854,0.993]$ & 90.9 & 72.7 & 100.0 \\
\hline Reader 1 & $0.771[0.546,0.904] *$ & 78.8 & 54.6 & 90.9 \\
\hline Reader 2 & $0.812[0.574,0.933]$ * & 81.8 & 72.7 & 86.4 \\
\hline
\end{tabular}

${ }^{*}$ Indicates a significant difference between deep learning and radiologists $(p<0.05)$.

\subsection{Diagnostic Performance of the Readers in the Test Set}

Figure 1 shows the receiver operating characteristic curves of the DL model and the radiologists' assessments. Significant differences in the AUCs at levels I-II (0.898 [DL] vs. 0.780 [R1] and 0.758 [R2]; both, $p<0.05)$ and level II ( 0.967 [DL] vs. 0.771 [R1] and 0.812 [R2]; both, $p<0.05$ ) between the DL model and the radiologists' assessments were found. The DL model was more accurate at levels I-II (85.9\% [DL] vs. 78.1\% [R1] and $78.1 \%$ [R2]), level I (80.6\% [DL] vs. $77.4 \%$ [R1] and 74.2\% [R2]), and level II (90.9\% [DL] vs. $78.8 \%$ [R1] and $81.4 \%$ [R2]). The DL model improved 16 diagnostic decisions of the readers. For the benign LNs at levels I-II, the DL model accurately diagnosed four and seven LNs that were misdiagnosed by R1 and R2, respectively, while one and two LNs that were accurately diagnosed by the readers were not accurately diagnosed by the DL model. For the metastatic LNs, the DL model improved four and one LNs that were diagnosed as benign lesions by R1 and R2, respectively, while two and one LNs that were accurately diagnosed by the readers were not accurately diagnosed by the DL model. A representative case of different diagnostic decisions between the DL model and radiologists is shown in Figure 2.
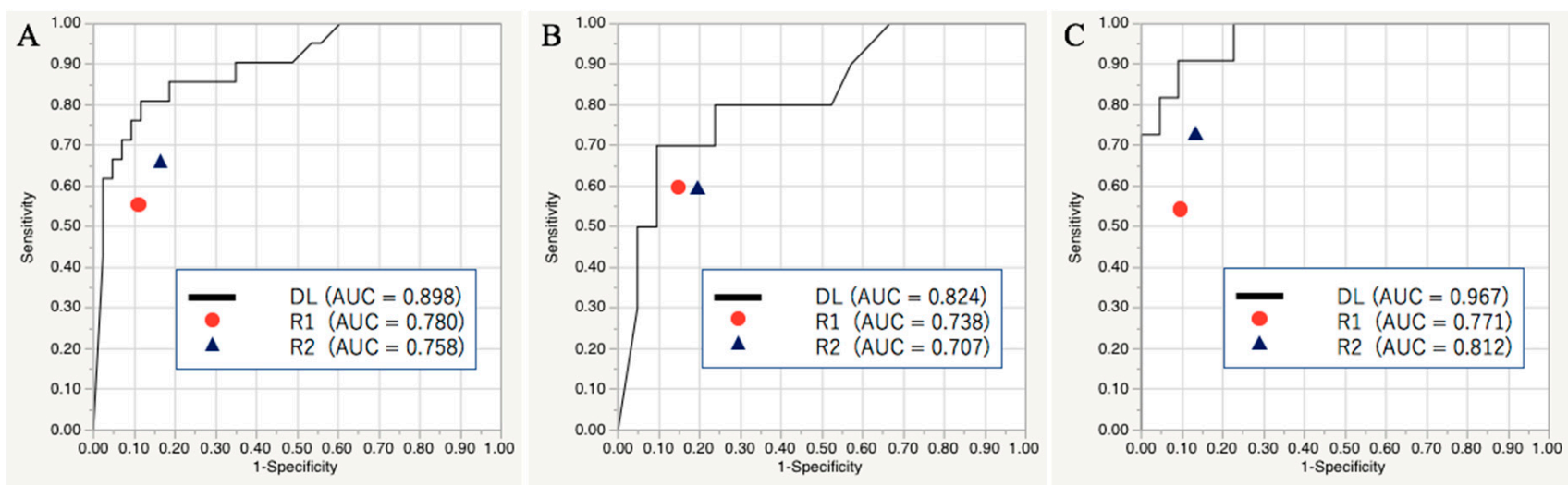

Figure 1. Areas under the receiver operating characteristic curves showing the deep learning model and radiologists' ability to identify metastatic cervical lymph nodes at levels I-II (A), I (B), and II (C) in the test set. 


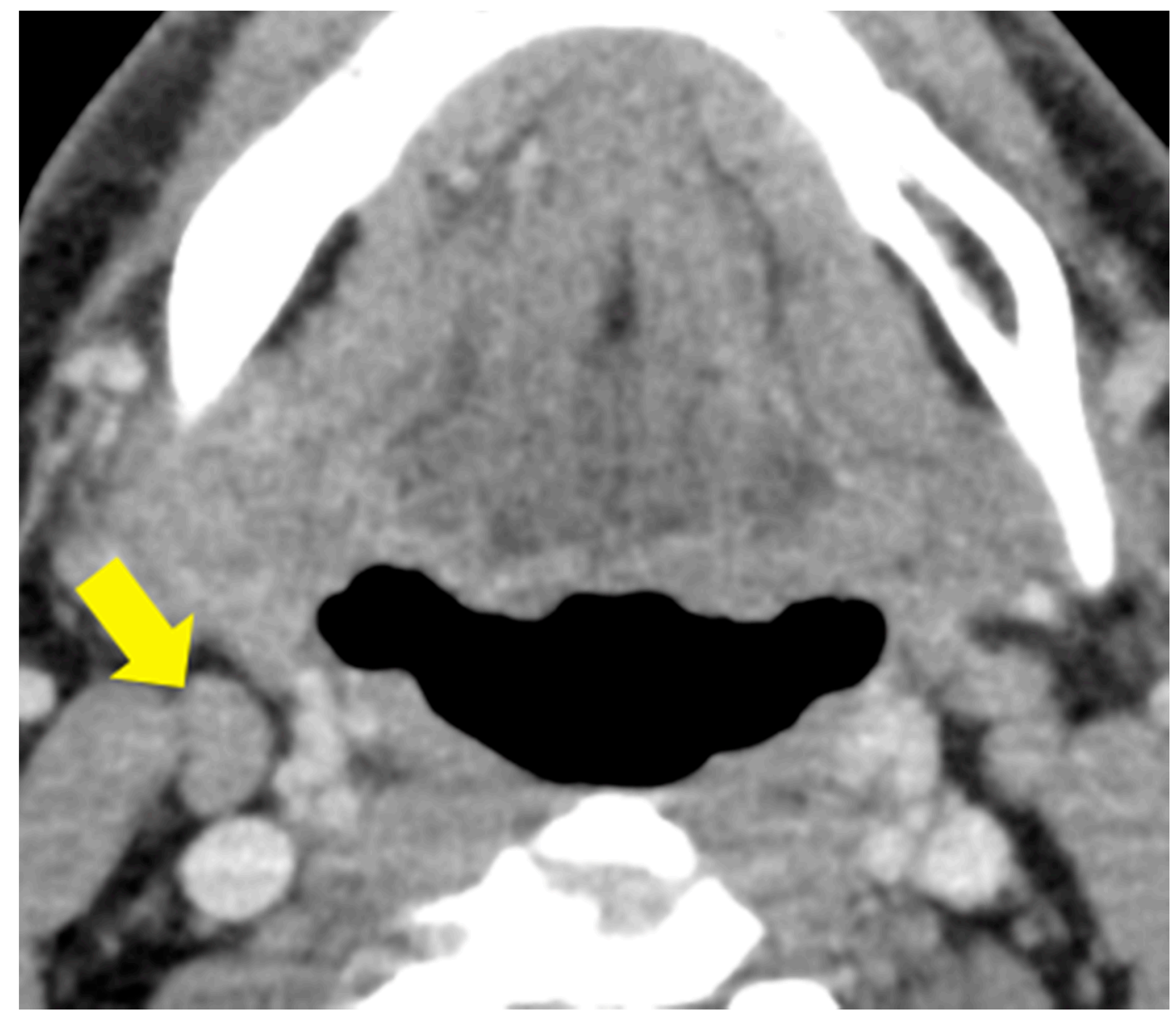

Figure 2. A representative case of cervical LN at level IIA on the right in a 56-year-old man with gingival cancer of T4 classification whose diagnosis differed between the deep learning (DL) model and radiologists' assessments. The targeted LN size was $8.2 \mathrm{~mm}$. The DL model diagnosed it as a metastatic LN, while two radiologists diagnosed it as a benign LN.

\section{Discussion}

In this study, the DL model achieved higher diagnostic performance in discriminating between benign and malignant cervical LNs on contrast-enhanced CT in patients with OSCC. In the test set, a significant difference in the AUCs of the DL models and radiologists was observed. Our results suggest that preoperative cervical nodal status at level I and II in patients with OSCC can be evaluated by DL.

The following CT and MR morphologic criteria have been widely used to determine the malignancy of cervical LNs in patients with head and neck cancer: Nodal size, peripheral shape, heterogeneous enhancement, and clustering of LNs. The diagnosis of LNs has depended on the judgment of radiologists and clinicians. Park et al. have reported that the sensitivity/specificity/accuracy of CT/MR for the visual assessment of cervical LNs in patients with head and neck SCC were $42 / 94 / 85 \%$ and $70 / 91 / 84 \%$ at the bilateral levels I and II, respectively [2].

Kann et al. [17] demonstrated that a test set evaluated using DualNet DL achieved a sensitivity/specificity/accuracy of 84/87/86\%, respectively. Similarly, an AUC of 0.91 for the assessment of the overall cervical LNs in head and neck cancer patients was found. However, no previous studies have reported the diagnostic performance of DL models at each LN level in OSCC patients. Our study can provide useful information about preoperative evaluation of cervical LN at levels I and II. In two studies, the entire LN was segmented for the assessment of the cervical LN status using CNNs [17,18]. However, in our study, the largest slice of the cervical LNs was used to simplify the workflow and avoid unnecessary CNN calculations. The center of the cervical LN can play a key role in its evaluation using 
CNNs. Ariji et al. [16] have described that DL with AlexNet could be useful in distinguishing benign and metastatic LNs from overall cervical LNs in OSCC patients. However, no significant difference between the DL model and the radiologists' assessments was found. Segmented CT images using an arbitrary-sized square included soft tissue structures around LNs in their study. Meanwhile, segmentation of the border of LNs without soft tissue structures was performed in our study. That might lead to improvement of the diagnostic performance using the DL model. However, these approaches of segmentation are not entirely automated and require human intervention. Fully automatic detection and classification of cervical LNs are required to improve the reproducibility. The targeted area of the cervical LN dissection should be precisely determined to minimize complications and the risk of residual tumor. For level-based analysis, especially at level I, small deposits of cancer cells that may not influence the appearance of the LN's internal architecture on CT can lead to false negatives. Thus, while CT exhibits high specificity for metastatic LN, it is not particularly sensitive. In our study, the accuracy of DL assessment of cervical LNs was superior to those of visual assessments. CNNs learn by reducing the differences between input and output data using backpropagation and loss function and identifying the useful connections within the neural network by itself. A large amount of excellent quality input data would allow high $\mathrm{CNN}$ performance. We utilized transfer learning using Xception in this study. In transfer leaning, the $\mathrm{CNN}$ architecture is pretrained from a large dataset, such as ImageNet, as the imaging features have already been extracted. Therefore, transfer learning improves the model's performance in limited datasets, and previous studies have utilized this approach for medical imaging [19-21]. Regarding showing a higher diagnostic performance of $\mathrm{CNN}$ compared with radiologists, $\mathrm{CNN}$ may have extracted some sort of image features that the radiologists could not recognize, which contributed to the discrimination between benign and metastatic cervical LNs.

There were several limitations to this study. First, selection bias was present, because patients who were suspected of having metastatic cervical LNs underwent dissection. Second, only a small number of LNs were used to create the DL model in this retrospective study. The cervical LNs at levels I and II were evaluated in the validation and test sets while LNs at levels I to V were included in the training set. Third, the image preprocessing protocol and DL model algorithm that we adopted might not be optimally suited for discriminating between benign and metastatic LNs since DL models for medical imaging are not yet sufficiently developed. Data volume and quality have a key role in improving the performance of DL models. Additionally, CT images that were acquired using two CT scanners were used. Although image standardization was performed, different image intensities originating from two scanners can affect the consistency of our results. For future studies, using the same CT scanner and protocol are preferable. Fourth, the diagnostic values of DL models have not been compared with those of PET-CT, which has widely spread as the best modality for the assessment of cervical LNs in head and neck cancers. The comparison leads to confirmation of the clinical significance of the DL models. Therefore, further large, multicenter studies are required to investigate the DL model with the optimal protocols for each level, compared with PET-CT. Fifth, there were seven patients who underwent dissection of their cervical LNs after the primary surgery. Postoperative inflammation might influence the LNs since cross sectional imaging for the assessment of recurrence is recommended after 2 to 3 months to avoid false lesions [22]. Sixth, eight cervical LN metastases were not identified on CT due to rapid growth. Hence, shortening the time between the CT examination and surgery is needed.

\section{Materials and Methods}

\subsection{Ethical Statement}

This retrospective study was approved by the Bioethics Committee of St. Marianna University School of Medicine (ethical code: 4469); the committee waived the requirement for informed consent due to the design of the study. All procedures were conducted according to the Declaration of Helsinki. 


\subsection{Subjects}

The study flowchart is shown in Figure 3. We reviewed our electronic medical records to identify patients with OSCC who underwent neck LN dissection and contrast-enhanced CT within 1 month before neck dissection between April 2013 and November 2017. The inclusion criteria were as follows: (1) Histopathologically confirmed OSCC (tongue cancer, gingival cancer, and floor of the mouth cancer); (2) histopathologically confirmed benign and metastatic cervical LNs at levels I-V; and (3) available preoperative CT data. The exclusion criteria were motion artifacts on CT $(n=1)$, preoperative chemotherapy $(n=2)$, and induction chemotherapy $(n=2)$. In total, 39 patients were enrolled in this study. The mean interval between cervical neck dissection and CT was $21.3 \pm 8.9$ days. Among 39 patients, 31 underwent primary resection and neck dissection and 7 underwent cervical neck dissection after primary resection based on the suspicion of metastatic cervical LNs. For the seven patients, the median interval between initial surgery and CT was 181 (range, 44-308) days.

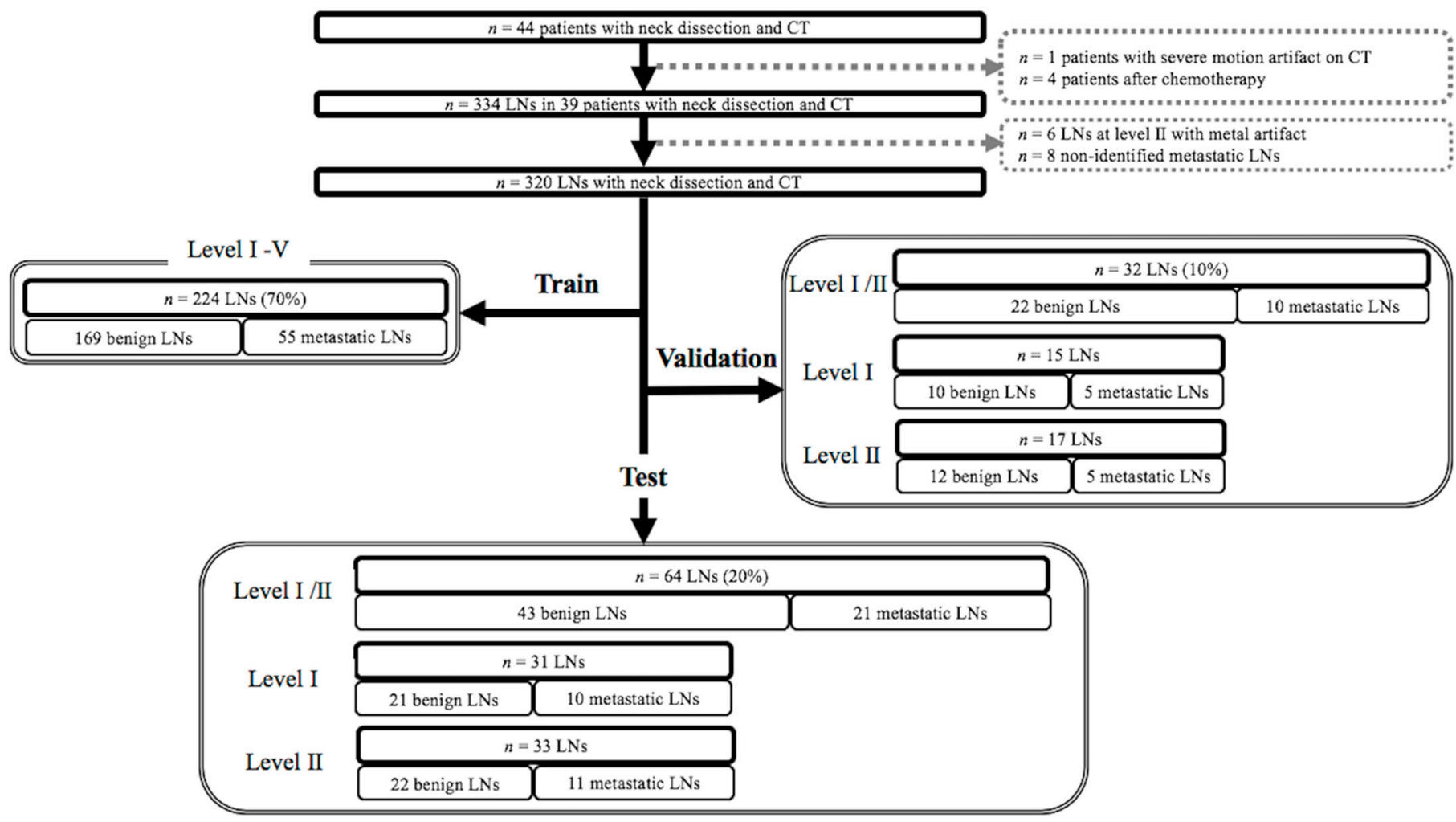

Figure 3. Flowchart of cervical lymph nodes included in the training, validation, and test sets.

\subsection{Computed Tomography}

CT from the base of the skull to the bottom of the neck was performed using 320-row scanners (Aquilion ONE; Canon Medical Systems, Otawara, Tochigi, Japan) for 23 patients and 64-row scanners (LightSpeed VCT; GE Healthcare, Milwaukee, WI, USA) for 16 patients according to the following protocols: For 320-row CT scanners: Collimation, $320 \times 0.5 \mathrm{~mm}$; tube voltage, $120 \mathrm{kVp}$; tube current, automatic exposure control; gantry rotation time, $0.5 \mathrm{~s}$; and beam pitch, 0.813. For 64-row CT scanners: Collimation, $64 \times 0.5 \mathrm{~mm}$; tube voltage, $120 \mathrm{kVp}$; tube current, automatic exposure control; gantry rotation time, $0.4 \mathrm{~s}$; and beam pitch, 0.984 . CT images with a 2-mm slice thickness without any overlap of serial sections were used. The imaging field of view was $230 \times 230 \mathrm{~mm}$. Iodine contrast material of $100 \mathrm{~mL}(300 \mathrm{mg} \mathrm{I} / \mathrm{mL})$ was intravenously injected at $1.5 \mathrm{~mL} / \mathrm{s}$ for both protocols. 


\subsection{Labeling of Cervical Lymph Nodes and Targeted Lymph Node}

Twenty-five patients underwent bilateral radical neck dissection, 11 underwent unilateral radical neck dissection, and 3 underwent unilateral supraomohyoid neck dissection. During surgery, the surgeon identified the cervical LNs for dissection using preoperative $\mathrm{CT}$ images. The operators set aside cervical LNs to determine their relative positions with reference to the size and location of LNs, vessels, muscles, salivary glands, and bones on these images. The dissected cervical LNs were stained with hematoxylin and eosin and evaluated by pathologists. LNs with histopathologically proven metastasis were labeled one-by-one at each level (levels I-IV). Initially, 334 cervical LNs were identified. However, six LNs were excluded because of severe metallic artifacts on CT images. Eight metastatic LNs were also excluded because they were not detected on CT owing to their rapid enlargement after performing CT. Therefore, 320 cervical LNs, comprising 234 benign and 86 metastatic LNs, at levels I-V were included in this study. We randomly categorized the cervical LNs into three sets: A training set at levels I-V $(n=224$ [70\%], 169 benign and 55 metastatic), a validation set at levels I-II ( $\mathrm{n}=32$ [10\%], 22 benign and 10 metastatic), and a test set at levels I-II ( $\mathrm{n}=64$ [20\%], 43 benign and 21 metastatic). In the validation and test sets, cervical LNs at levels III-V were not used because the necessary sample sizes for each level, as mentioned in the "statistical analysis" section, were unavailable, which could weaken the statistical power.

\subsection{Image Preprocessing for Deep Learning}

The study workflow is shown in Figure 4. Three CT images, namely the image showing the largest cross-sectional area of the targeted LN and the adjacent images (one cranial and one caudal image), were obtained using OsirixMD software (Pixmeo, Bernex, Switzerland). The margin of the LNs on the selected images were contoured as close as possible by a single radiologist (**blinded ${ }^{* *}$ with 9 years of experience).

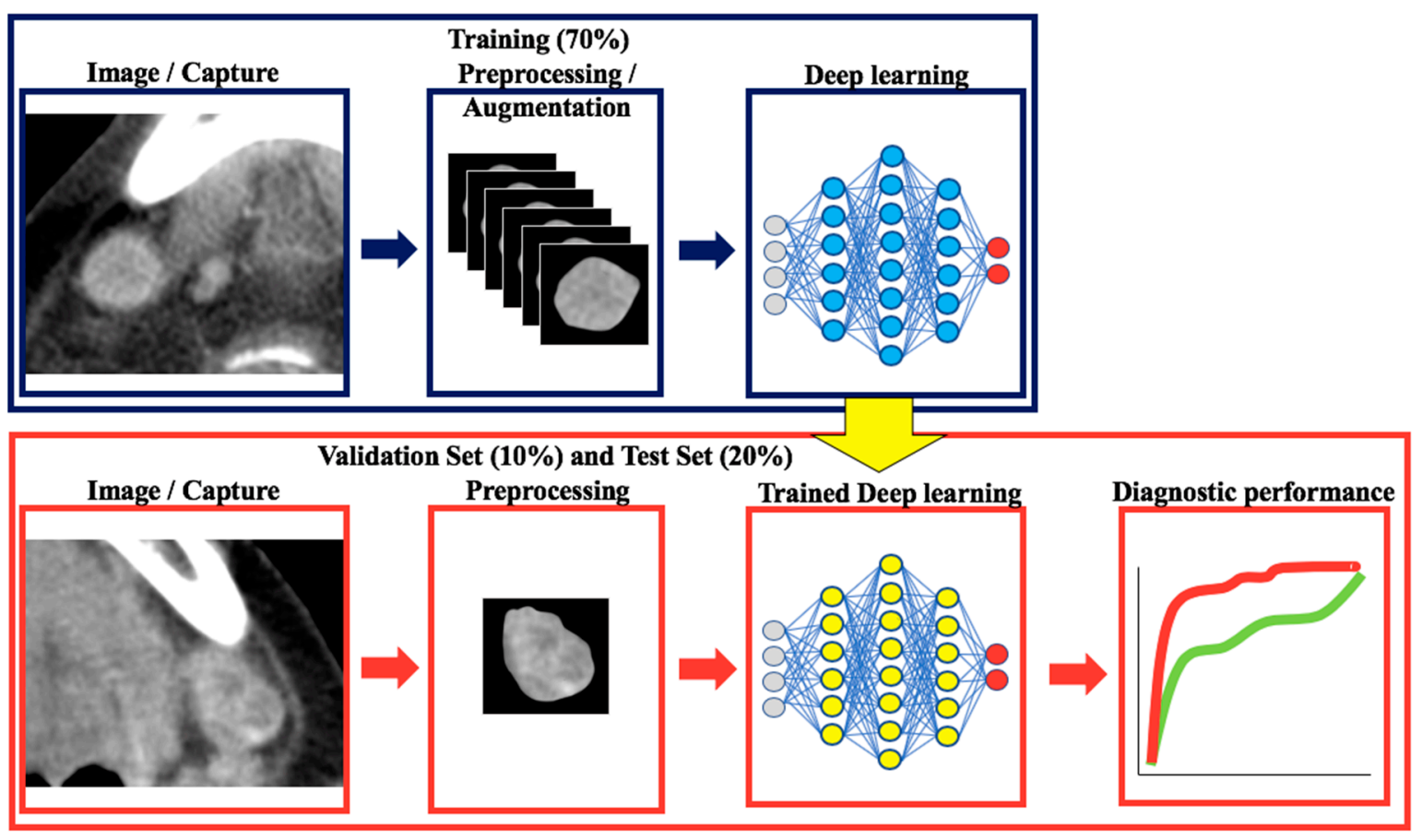

Figure 4. Workflow of this study.

All images were resized to $300 \times 300$ pixels. All images were normalized and divided by 255 before the augmentation. The resized images were augmented by horizontal flip, vertical flip, width shift, and height shift. The programming language used for augmentation was Python 3.6 (https:/ / www.python.org). 


\subsection{Classification with Convolutional Neural Networks and Transfer Learning}

In this study, the network architecture was based on the Xception architecture [23]. This network comprised three flows, namely entry flow, middle flow, and exit flow. Each flow is composed of several modules called Inception, which is a component of GoogleNet [24]. A detailed description of the Xception architecture is given in Appendix A (Figure A1). For our experiment, we used the Xception architecture pretrained on the ImageNet dataset. Only the Exit flow of the network was fine tuned to our dataset to classify benign and metastatic cervical LNs. Early stopping was conducted to avoid overfitting in the training set. This method stops training without fixing the number of epochs when validation loss is confirmed. For the validation and test sets, the performance of the trained DL model was evaluated. In the test set, to match the DL model and visual assessment findings, the largest slice of the cervical LN was used for the final analysis.

\subsection{Visual Analysis}

The interpretation of $\mathrm{CT}$ images was based on visual assessment by two board-certified radiologists (R1 and R2, with 9 and 19 years of experience reading head and neck $\mathrm{CT}$, respectively) who were blinded to patients' clinical information, including histopathological results. Both radiologists evaluated the cervical LNs and graded them using a 4-point scale: 1 = definitely benign; 2 = likely benign; $3=$ likely metastatic; and $4=$ definitely metastatic. The following CT characteristics were considered to judge the scale: Shortest maximum diameter of more than $11 \mathrm{~mm}$ in the jugulo-digastric area and $10 \mathrm{~mm}$ in other cervical areas, heterogeneous enhancement or central necrosis, or loss of fatty hilum [2,3].

\subsection{Statistical Analysis}

The necessary number of LNs was calculated to evaluate the area under the curve (AUC) with a type I error of $5 \%$ and power of $80 \%$ using the R statistical package (version 3.6.1; R Project for Statistical Computing, R Foundation, Vienna, Austria). A previous study had reported an AUC of 0.801 in quantitative detection of metastatic cervical LNs in patients with OSCC [25]. Our training cohort showed a benign to metastatic LN ratio of 3:1. We estimated that a sample size of at least 27 was required.

Statistical analysis was performed using Python 3.6 or JMP pro 14.2.0 software (SAS Institute, Cary, NC, USA). In the test set, sensitivities, specificities, diagnostic accuracy rates, and AUCs of the DL model and the radiologists' assessments were analyzed to determine their ability to differentiate between benign and metastatic cervical LNs at levels I-II, I, and II. The AUCs were compared between the DL model and the radiologists' assessments. $p$-values $<0.05$ were considered to indicate a statistically significant difference.

\section{Conclusions}

In conclusion, DL can differentiate between benign and metastatic cervical LNs on preoperative contrast-enhanced CT of patients with OSCC, which can help guide treatment decisions on neck dissection in a reproducible manner. Further investigation will be required to establish the optimal diagnostic method for cervical LN status.

Author Contributions: Conceptualization, H.T. and J.H.; methodology, H.T.; validation, H.T., T.Y., T.K., S.M. (Sono Mishiro), D.H., and E.T.; formal analysis, H.T., T.K., S.M. (Sono Mishiro), D.H., and E.T.; investigation, H.T., T.Y., T.K., S.M. (Sono Mishiro), D.H., and E.T.; data curation, H.T., J.H., and T.N.; writing—original draft preparation, H.T. and E.T.; writing-review \& editing, T.Y., J.H., T.N., H.M., S.M. (Sadayuki Murayama), and Y.K.; supervision, Y.K. All authors have read and agreed to the published version of the manuscript.

Funding: This research was supported by the grants from St. Marianna University School of Medicine and Daiichi Sankyo grant program (There are no specific codes/numbers).

Institutional Review Board Statement: This retrospective study was approved by the Bioethics Committee of St. Marianna University School of Medicine (ethical code: 4469). All procedures were conducted according to the Declaration of Helsinki. 
Informed Consent Statement: The committee waived the requirement for informed consent due to the design of the study.

Data Availability Statement: Most of the data in this study are available in this article. Some data including CT imagings are not publicly available due to data protection regulations.

Conflicts of Interest: The authors declare no conflict of interest.

\section{Appendix A}
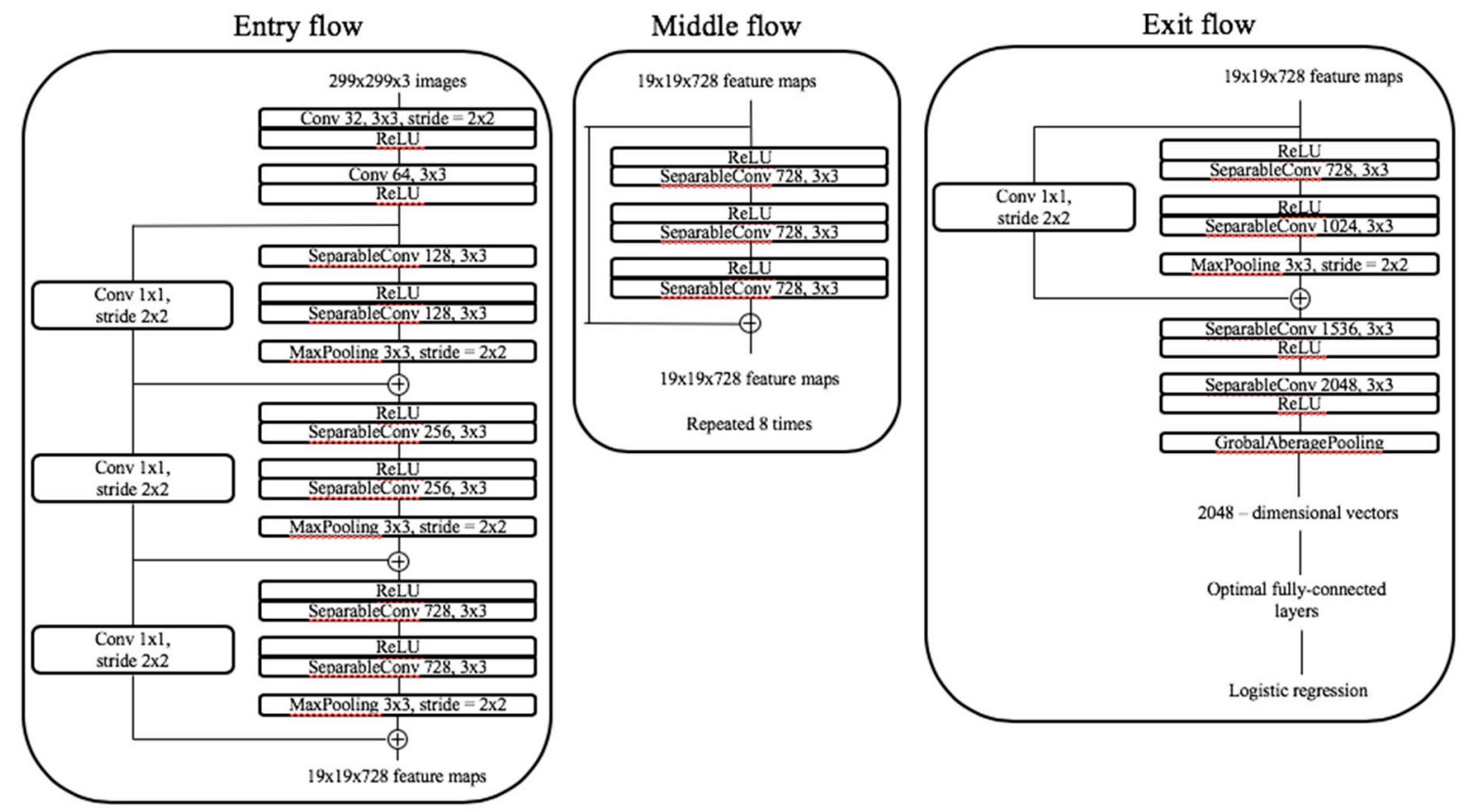

Figure A1. The architecture of Xception model.

\section{References}

1. Roh, J.L.; Park, J.P.; Kim, J.S.; Lee, J.H.; Cho, K.J.; Choi, S.H.; Nam, S.Y.; Kim, S.Y. 18F Fluorodeoxyglucose PET/CT in Head and Neck Squamous Cell Carcinoma with Negative Neck Palpation Findings: A Prospective Study. Radiology 2014, $271,153-161$. [CrossRef] [PubMed]

2. $\quad$ Park, J.T.; Roh, J.L.; Kim, J.S.; Lee, J.H.; Cho, K.J.; Choi, S.H.; Nam, S.Y.; Kim, S.Y. (18)F FDG PET/CT Versus CT/MR Imaging and the Prognostic Value of Contralateral Neck Metastases in Patients with Head and Neck Squamous Cell Carcinoma. Radiology 2016, 279, 481-491. [CrossRef]

3. Shin, N.Y.; Lee, J.H.; Kang, W.J.; Koh, Y.W.; Sohn, B.; Kim, J. Clinical Usefulness of [18F]FDG PET-CT and CT/MRI for Detecting Nodal Metastasis in Patients with Hypopharyngeal Squamous Cell Carcinoma. Ann. Surg. Oncol. 2015, 22, 994-999. [CrossRef]

4. Sumi, M.; Ohki, M.; Nakamura, T. Comparison of Sonography and CT for Differentiating Benign from Malignant Cervical Lymph Nodes in Patients with Squamous Cell Carcinoma of the Head and Neck. Am. J. Roentgenol. 2001, 176, 1019-1024. [CrossRef]

5. Foust, A.M.; Ali, R.M.; Nguyen, X.V.; Agrawal, A.; Prevedello, L.M.; Bourekas, E.C.; Boulter, D.J. Dual-Energy CT-Derived Iodine Content and Spectral Attenuation Analysis of Metastatic Versus Nonmetastatic Lymph Nodes in Squamous Cell Carcinoma of the Oropharynx. Tomography 2018, 4, 66-71.

6. Liu, X.; Ouyang, D.; Li, H.; Zhang, R.; Lv, Y.; Yang, A.; Xie, C. Papillary Thyroid Cancer: Dual-Energy Spectral CT Quantitative Parameters for Preoperative Diagnosis of Metastasis to the Cervical Lymph Nodes. Radiology 2015, 275, 167-176. [CrossRef]

7. Takamochi, K.; Yoshida, J.; Murakami, K.; Niho, S.; Ishii, G.; Nishimura, M.; Nishiwaki, Y.; Suzuki, K.; Nagai, K. Pitfalls in Lymph Node Staging with Positron Emission Tomography in Non-Small Cell Lung Cancer Patients. Lung Cancer 2005, 47, 235-242. [CrossRef]

8. Yamazaki, Y.; Saitoh, M.; Notani, K.; Tei, K.; Totsuka, Y.; Takinami, S.; Kanegae, K.; Inubushi, M.; Tamaki, N.; Kitagawa, Y. Assessment of Cervical Lymph Node Metastases using FDG-PET in Patients with Head and Neck Cancer. Ann. Nucl. Med. 2008, 22, 177-184. [CrossRef]

9. Mahieu, R.; de Maar, J.S.; Nieuwenhuis, E.R.; Deckers, R.; Moonen, C.; Alic, L.; Ten Haken, B.; de Keizer, B.; Bree, R. New Developments in Imaging for Sentinel Lymph Node Biopsy in Early-Stage Oral Cavity Squamous Cell Carcinoma. Cancers 2020, 12, 3055. [CrossRef] 
10. den Toom, I.J.; Boeve, K.; Lobeek, D.; Bloemena, E.; Donswijk, M.L.; de Keizer, B.; Klop, W.M.C.; Leemans, C.R.; Willems, S.M.; Takes, R.P.; et al. Elective Neck Dissection Or Sentinel Lymph Node Biopsy in Early Stage Oral Cavity Cancer Patients: The Dutch Experience. Cancers 2020, 12, 1783. [CrossRef]

11. Yanagawa, M.; Niioka, H.; Hata, A.; Kikuchi, N.; Honda, O.; Kurakami, H.; Morii, E.; Noguchi, M.; Watanabe, Y.; Miyake, J.; et al. Application of Deep Learning (3-Dimensional Convolutional Neural Network) for the Prediction of Pathological Invasiveness in Lung Adenocarcinoma: A Preliminary Study. Medicine 2019, 98, e16119. [CrossRef]

12. Peng, J.; Kang, S.; Ning, Z.; Deng, H.; Shen, J.; Xu, Y.; Zhang, J.; Zhao, W.; Li, X.; Gong, W.; et al. Residual Convolutional Neural Network for Predicting Response of Transarterial Chemoembolization in Hepatocellular Carcinoma from CT Imaging. Eur. Radiol. 2019, 30, 413-424. [CrossRef]

13. Yasaka, K.; Akai, H.; Abe, O.; Kiryu, S. Deep Learning with Convolutional Neural Network for Differentiation of Liver Masses at Dynamic Contrast-Enhanced CT: A Preliminary Study. Radiology 2018, 286, 887-896. [CrossRef]

14. Aldoj, N.; Lukas, S.; Dewey, M.; Penzkofer, T. Semi-Automatic Classification of Prostate Cancer on Multi-Parametric MR Imaging using a Multi-Channel 3D Convolutional Neural Network. Eur. Radiol. 2019, 30, 1243-1253. [CrossRef]

15. Zhuge, Y.; Krauze, A.V.; Ning, H.; Cheng, J.Y.; Arora, B.C.; Camphausen, K.; Miller, R.W. Brain Tumor Segmentation using Holistically Nested Neural Networks in MRI Images. Med. Phys. 2017, 44, 5234-5243. [CrossRef] [PubMed]

16. Ariji, Y.; Fukuda, M.; Kise, Y.; Nozawa, M.; Yanashita, Y.; Fujita, H.; Katsumata, A.; Ariji, E. Contrast-Enhanced Computed Tomography Image Assessment of Cervical Lymph Node Metastasis in Patients with Oral Cancer by using a Deep Learning System of Artificial Intelligence. Oral Surg. Oral Med. Oral Pathol. Oral Radiol. 2019, 127, 458-463. [CrossRef]

17. Kann, B.H.; Aneja, S.; Loganadane, G.V.; Kelly, J.R.; Smith, S.M.; Decker, R.H.; Yu, J.B.; Park, H.S.; Yarbrough, W.G.; Malhotra, A.; et al. Pretreatment Identification of Head and Neck Cancer Nodal Metastasis and Extranodal Extension using Deep Learning Neural Networks. Sci. Rep. 2018, 8, 1-11. [CrossRef]

18. Kann, B.H.; Hicks, D.F.; Payabvash, S.; Mahajan, A.; Du, J.; Gupta, V.; Park, H.S.; Yu, J.B.; Yarbrough, W.G.; Burtness, B.A.; et al. Multi-Institutional Validation of Deep Learning for Pretreatment Identification of Extranodal Extension in Head and Neck Squamous Cell Carcinoma. J. Clin. Oncol. 2019, 38, 1304-1311. [CrossRef] [PubMed]

19. Chen, H.; Ni, D.; Qin, J.; Li, S.; Yang, X.; Wang, T.; Heng, P.A. Standard Plane Localization in Fetal Ultrasound Via Domain Transferred Deep Neural Networks. IEEE J. Biomed. Health. Inform. 2015, 19, 1627-1636. [CrossRef] [PubMed]

20. Ariji, Y.; Sugita, Y.; Nagao, T.; Nakayama, A.; Fukuda, M.; Kise, Y.; Nozawa, M.; Nishiyama, M.; Katumata, A.; Ariji, E. CT Evaluation of Extranodal Extension of Cervical Lymph Node Metastases in Patients with Oral Squamous Cell Carcinoma using Deep Learning Classification. Oral Radiol. 2020, 36, 148-155. [CrossRef] [PubMed]

21. Diaz-Pinto, A.; Morales, S.; Naranjo, V.; Kohler, T.; Mossi, J.M.; Navea, A. CNNs for Automatic Glaucoma Assessment using Fundus Images: An Extensive Validation. Biomed. Eng. Online 2019, 18, 1-19. [CrossRef] [PubMed]

22. Dutta, P.R.; Riaz, N.; McBride, S.; Morris, L.G.; Patel, S.; Ganly, I.; Wong, R.J.; Palmer, F.; Schoder, H.; Lee, N. Postoperative PET/CT and Target Delineation before Adjuvant Radiotherapy in Patients with Oral Cavity Squamous Cell Carcinoma. Head Neck 2016, 38 (Suppl. 1), E1285-E1293. [CrossRef] [PubMed]

23. Chollet, F. Xception: Deep Learning with Depthwise Separable Convolutions. In Proceedings of the IEEE Conference on Computer Vision and Pattern Recognition, Honolulu, HI, USA, 21-26 July 2017; pp. 1251-1258.

24. Szegedy, C.; Liu, W.; Jia, Y.; Sermanet, P.; Reed, S.; Anguelov, D.; Erhan, D.; Vanhoucke, V.; Rabinovich, A. Going Deeper with Convolutions. In Proceedings of the IEEE Conference on Computer Vision and Pattern Recognition, Boston, MA, USA, 7-12 June 2015; pp. 1-9.

25. Ng, S.H.; Yen, T.C.; Liao, C.T.; Chang, J.T.; Chan, S.C.; Ko, S.F.; Wang, H.M.; Wong, H.F. 18F-FDG PET and CT/MRI in Oral Cavity Squamous Cell Carcinoma: A Prospective Study of 124 Patients with Histologic Correlation. J. Nucl. Med. 2005, 46, 1136-1143. [PubMed] 